\title{
PAPER \\ Noncontact Monitoring of Heartbeat and Movements during Sleep Using a Pair of Millimeter-Wave Ultra-Wideband Radar Systems
}

\author{
Takuya SAKAMOTO $^{\dagger, \dagger \dagger a)}$, Member, Sohei MITANI $^{\dagger \dagger}$, Nonmember, and Toru SATO $^{\dagger \dagger \dagger}$, Fellow
}

\begin{abstract}
SUMMARY We experimentally evaluate the performance of a noncontact system that measures the heartbeat of a sleeping person. The proposed system comprises a pair of radar systems installed at two different positions. We use millimeter-wave ultra-wideband multiple-input multiple-output array radar systems and evaluate the performance attained in measuring the heart inter-beat interval and body movement. The importance of using two radar systems instead of one is demonstrated in this paper. We conduct three types of experiments; the first and second experiments are radar measurements of three participants lying on a bed with and without body movement, while the third experiment is the radar measurement of a participant actually sleeping overnight. The experiments demonstrate that the performance of the radar-based vital measurement strongly depends on the orientation of the person under test. They also show that the proposed system detects $70 \%$ of rolling-over movements made overnight.

key words: vital signs, ultra-wideband radar, body movement
\end{abstract}

\section{Introduction}

Since the outbreak of COVID-19, it has been widely recognized that the outbreak of new infectious diseases can result in a shortage of medical resources. It is thus necessary for some patients to be followed up at home or isolation facilities rather than medical institutions. To carry out such followup observations safely, it is essential to record the patients' vital signs, such as respiration and heartbeat signs, over a long period of time. Contact sensors, such as those adopted in electrocardiography (ECG) and photoplethysmography, have been widely used to monitor vital signs. However, the long-term wearing of such sensors can cause discomfort and skin inflammation.

Noncontact radar sensors are therefore preferable for the long-term monitoring of people. In particular, microwaves and millimeter waves (MMWs) are able to penetrate clothing and comforters well, allowing the measurement of a small displacement of the target person associated with a vital sign. The authors previously developed an algorithm referred to as the topology method [1], [2], which estimates heart inter-beat

\footnotetext{
Manuscript received May 23, 2020.

Manuscript revised September 2, 2020.

Manuscript publicized October 6, 2020.

${ }^{\dagger}$ The author is with the Graduate School of Engineering, Kyoto University, Kyoto-shi, 615-8510 Japan.

${ }^{\dagger}$ The author is with PRESTO, Japan Science and Technology Agency, Kawaguchi-shi, 332-0012 Japan.

${ }^{\dagger \dagger}$ The author was with the Graduate School of Informatics, Kyoto University, Kyoto-shi, 606-8501 Japan.

$+1+\dagger$ The author is with the Institute for Liberal Arts and Sciences, Kyoto University, Kyoto-shi, 606-8501 Japan.

a) E-mail: sakamoto.takuya.8n@kyoto-u.ac.jp

DOI: $10.1587 /$ transcom.2020EBP3078
}

intervals (IBIs) accurately from a radar signal. The topology method extracts feature (local maximum, minimum, and inflection) points from a radar echo phase. Using the sequence pattern of the feature points, unreliable estimate points are rejected, which improves the overall accuracy in estimating the heart IBI.

Most studies on measuring seated individuals place the radar system in front of the chest or abdomen of the person [3]-[12]. This is partly because the studies intended to measure respiration and the heartbeat at the same time. Nagae and Mase [13] measured the leg of a seated person. The present authors measured the soles [14] and the top of the head [15]. Vinci et al. [16] measured the front and back sides of the torso for comparison. Wang et al. [17] and Tang et al. [18] measured the front and back sides of the torso simultaneously to cancel out body movements. Li et al. [19] and Wang et al. [20] both measured four sides (front, back, left, and right) of the torso for comparison.

When measuring a sleeping person, where should we install radar antennas? In existing studies on radar-based sleep monitoring, antennas were installed directly above the bed [16], [21]-[25], diagonally above the bed [26]-[29], next to the bed [30]-[33], and under the bed or mattress [34]-[37]. We note that in sleep monitoring, changes in body orientation and movement cannot be neglected.

In this paper, we demonstrate the benefit of using multiple radar systems in monitoring vital signs and body movements of a sleeping person. We develop a measurement system comprising a pair of MMW ultra-wideband (UWB) multiple-input multiple-output (MIMO) array radar systems and conduct three types of measurement to evaluate the performance of the system. We first conduct an experiment to measure the chest and shoulder of a target person lying without movement on a bed. The accuracy in estimating the heart IBI is carefully evaluated to clarify the effect of the position of radar systems. We next conduct an experiment with a person lying with body movement to evaluate the effect of the motion on accuracy. We finally conduct an experiment with a person sleeping in a bed overnight. The novelty of the present paper is that it is the first to report the benefit of using multiple radar systems to observe a target person from different angles for noncontact sleep monitoring. In addition, the present paper demonstrates the effect of the body part to be measured in radar-based sleep monitoring. 


\section{Radar System and Measurement Setup}

Participants lying in a bed were measured using a pair of radar systems installed at two locations. Figure 1 shows the front side of the radar module. The individual antennas in the array are called elements. Here, we see four horizontally aligned transmit elements (Tx) and four vertically aligned receive elements $(\mathrm{Rx})$. In this study, we used only two transmitting elements - Tx 1 and $\mathrm{Tx} 2-$ and two receiving elements - Rx1 and $\mathrm{R} \times 2$ - among the eight elements, resulting in $2 \times 2=4$ channels in total. The central frequency and bandwidth of the system were respectively 79.0 and $2.0 \mathrm{GHz}$, corresponding to a central wavelength of $3.80 \mathrm{~mm}$ and a range resolution of $75.0 \mathrm{~mm}$. The slow-time sampling interval was $0.238 \mathrm{~ms}$. The antenna beam width was $90^{\circ}$. All element spacings were $2.263 \mathrm{~mm}$, corresponding to 0.60 wavelengths. A block diagram of the radar system is shown in Fig. 2. The phase-locked loop (PLL) generated a signal with a frequency of $15.8 \mathrm{GHz}$, which was then multiplied by a factor of 5 by a sub-harmonic injection locked oscillator (SH-ILO), producing a 79-GHz carrier signal. The 79-GHz signal was phase-modulated with a binary $\mathrm{m}$-sequence generated by a pseudo random noise (PRN) code generator. The $\mathrm{m}$-sequence was 511 samples long and had a chip rate of $2.0 \mathrm{Gcps}$, which corresponds to an inter-pulse period of $255.5 \mathrm{~ns}$ after pulse compression, resulting in a maximum target range of $38.3 \mathrm{~m}$. The signal was transmitted from transmitting antennas. The echoes reflected by targets were received by receiving elements, fed to IQ mixers, and A/D converted. Finally, the digital signals were demodulated using the same m-sequence. In an evaluation of the accuracy in estimating the heart IBI, the participants wore an ECG15102017 contact ECG sensor (PLUX Wireless Biosignals S.A., Lisboa, Portugal), whose sampling frequency was $500 \mathrm{~Hz}$. Figure 3 shows the measurement setup with a participant in a supine position in a bed. Radar 1 was installed vertically above the chest while radar 2 was installed laterally at the height of the participant's left shoulder. The distances between a participant and radars 1 and 2 were approximately 1.7 and $1.2 \mathrm{~m}$, respectively. The bed height was $0.6 \mathrm{~m}$ and the heights of radars 1 and 2 from the floor were 2.5 and $0.7 \mathrm{~m}$, respectively. This experimental setting is only an example. Although it is important to optimize settings including the radar positions, we leave this to future work.

\section{Vital Monitoring and Body Motion Detection}

Signal $s_{i, j}(t, r)$ is defined as a complex-valued signal that is transmitted from the $i$-th transmitting element and received at the $j$-th receiving element $(i, j=1,2)$, where $t$ is slow time and the range $r=c t_{\mathrm{d}} / 2$ is expressed using the speed of light $c$ and delay time $t_{\mathrm{d}}$. We apply Hu's circle fitting method [8], [38] to the signal trajectory in the complex plane to estimate static clutter $\tilde{s}_{i, j}$. The estimated clutter $\tilde{s}_{i, j}$ is subtracted from the signal to obtain the time-varying component $\tilde{s}_{i, j}(t)=$ $s_{i, j}\left(t, r_{0}\right)-\tilde{s}_{i, j}$, where $r_{0}$ is the estimated range.

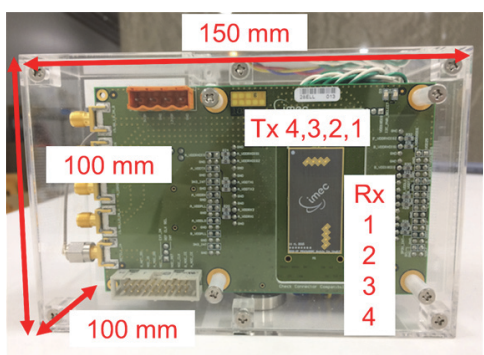

Fig. 1 MMW UWB MIMO array radar module with four transmitting and four receiving elements.

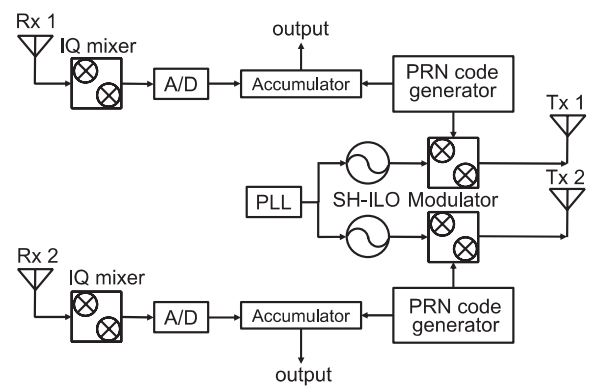

Fig. 2 Block diagram of the MMW UWB MIMO array radar.

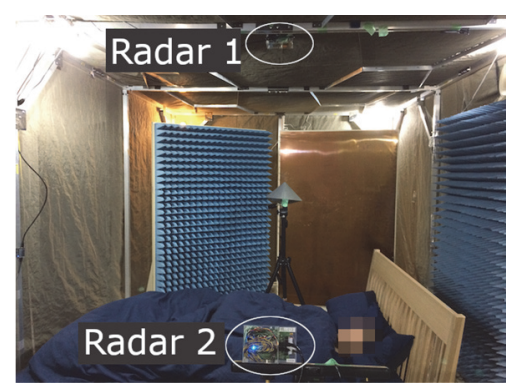

Fig. 3 Measurement setup with a pair of radar systems and a participant lying in a bed.

The vector $\boldsymbol{s}=\left[\tilde{s}_{1,1}, \tilde{s}_{1,2}, \tilde{s}_{2,1}, \tilde{s}_{2,2}\right]^{\mathrm{T}}$ is then formed and its correlation matrix $R_{s s}=\left\langle\boldsymbol{s} \boldsymbol{s}^{\mathrm{H}}\right\rangle$ is obtained, where superscripts $\mathrm{T}$ and $\mathrm{H}$ respectively denote the transpose and complex-conjugate transpose. The largest eigenvalue $\sigma_{\max }$ of $R_{s s}$ and its corresponding eigenvector $\boldsymbol{v}_{\max }$ are calculated, and the maximum ratio combining technique [15], [33] is then applied to obtain the body displacement as $d(t)=(\lambda / 4 \pi)$ unwrap $\left(\angle \boldsymbol{v}_{\max }^{\mathrm{H}} \boldsymbol{s}(t)\right)$, where $\angle$ denotes the phase of a complex value while unwrap $(\cdot)$ denotes an unwrapping process that obtains a smooth phase sequence considering the phase ambiguity. The unwrapping process corrects the phase by adding multiples of $\pm 2 \pi$ when the absolute jump between adjacent samples is greater than $\pi$ so that the resultant phase sequence becomes smooth.

Body motion is detected according to the following procedure. We define a binary-valued function $f_{\mathrm{m}}(\tau)$ such that $f_{\mathrm{m}}(\tau)=1$ if a body motion is detected at time $\tau$ and $f_{\mathrm{m}}(\tau)=0$ otherwise.

Step 1) Initialize time $\tau \leftarrow 0$. 
Step 2) If a displacement change over time length $T_{0}$ is greater than a threshold $d_{\text {th }}$ (i.e., $\left|d(\tau)-d\left(\tau-T_{0}\right)\right| \geq$ $\left.d_{\text {th }}\right)$, we set $f_{\mathrm{m}}(\tau)=1$, which means that motion is detected. Otherwise, we set $f_{\mathrm{m}}(\tau)=0$.

Step 3) If $f_{\mathrm{m}}(\tau)=0$ (i.e., no body motion is detected), the range $r_{0}(\tau)=r_{0}\left(\tau-T_{0}\right)$ is not updated, and the topology method [1] is applied to the signal at range $r_{0}$ to obtain the IBI.

If $f_{\mathrm{m}}(\tau)=1$, the target range $r_{0}$ is estimated as $r_{0}(\tau)=\arg \max _{r} \sum_{i, j} \int_{\tau-T}^{\tau}\left|s_{i, j}(t, r)-\hat{s}_{i, j}(\tau, r)\right|^{2} \mathrm{~d} t$, where $\hat{s}_{i, j}(\tau, r)=\frac{1}{T} \int_{\tau-T}^{\tau} s_{i, j}\left(t^{\prime}, r\right) \mathrm{d} t^{\prime}$.

Step 4) $\quad \tau$ is updated as $\tau \leftarrow \tau+T_{0}$ and we return to Step 2).

In this paper, parameters are set empirically as $T=6 \mathrm{~s}$, $T_{0}=0.6 \mathrm{~s}$, and $d_{\mathrm{th}}=30 \mathrm{~mm}$

\section{Performance Indices of Noncontact Vital Monitoring}

We use three indices, namely the time coverage rate (TCR) $\rho_{\mathrm{t}}$, acquisition rate (AR) $\rho_{\mathrm{a}}$, and selective root mean square error (RMSE), in evaluating the performance of a radar measurement of vital signs. We assume that heart IBIs measured in ECG are sufficiently accurate and use them as a reference to evaluate estimation errors in the following definitions.

\subsection{Time Coverage Rate (TCR)}

First, the total measurement time $T_{\text {all }}$ is equally divided into $N$ intervals, all having lengths $\Delta t$. Let us assume that among a total of $N$ intervals, there are $n$ intervals that contain at least one accurate estimate point with error of less than $\varepsilon$. We define the time coverage rate $\rho_{\mathrm{t}}=n / N=n \Delta t / T_{\text {all }}$. In this paper, we set $\Delta t=1 \mathrm{~s}$ and $\varepsilon=50 \mathrm{~ms}$ empirically. If accurate estimate points are distributed uniformly, the TCR is high even if there are numerous inaccurate estimate points. By contrast, even if there are numerous accurate estimate points, the TCR is low if the estimate points are concentrated within a narrow time interval.

\subsection{Acquisition Rate (AR)}

Let us assume that there are $M_{\text {all }}$ estimate points, and among them, there are $m$ estimate points whose error is less than $\varepsilon$. The $\operatorname{AR} \rho_{\mathrm{a}}$ is then given by $\rho_{\mathrm{a}}=m / M_{\mathrm{all}}$, which is the ratio of accurate estimates to all estimates. The AR indicates the reliability of the estimates; even if there are numerous accurate estimates, the $\mathrm{AR}$ is low if there are equally numerous inaccurate estimates as well. When the TCR is low, the TCR can be increased appreciably by adding just one more accurate estimate point, whereas the AR is stable and hardly affected by the addition of such a single point. The TCR and AR should thus be used together to evaluate performance objectively.

\subsection{Selective Root Mean Square Error (RMSE)}

If an error becomes large temporarily owing to an inappro-
Table 1 Accuracy of the heart IBI for three participants.

\begin{tabular}{|c|c|c|c|c|}
\hline Subject & Body Part & TCR & AR & RMSE \\
\hline \multirow{2}{*}{ A } & Chest & $68.7 \%$ & $58.8 \%$ & $21.7 \mathrm{~ms}$ \\
\cline { 2 - 5 } & Shoulder & $80.0 \%$ & $74.8 \%$ & $24.0 \mathrm{~ms}$ \\
\hline \multirow{2}{*}{ B } & Chest & $43.3 \%$ & $31.3 \%$ & $31.2 \mathrm{~ms}$ \\
\cline { 2 - 5 } & Shoulder & $53.3 \%$ & $31.2 \%$ & $22.2 \mathrm{~ms}$ \\
\hline \multirow{2}{*}{ C } & Chest & $42.7 \%$ & $26.1 \%$ & $33.1 \mathrm{~ms}$ \\
\cline { 2 - 5 } & Shoulder & $73.3 \%$ & $57.0 \%$ & $28.4 \mathrm{~ms}$ \\
\hline
\end{tabular}

priate orientation of the target person, the temporary increase in the error can be evaluated using the TCR. The RMSE in this paper is therefore calculated using only accurate estimate points whose error is less than $\varepsilon$.

\section{Experiment with a Person Lying without Body Mo- tion}

First, to clarify which body part of a sleeping person is suitable for the radar measurement of vital signs, we measured the chests and shoulders of three participants lying in the supine position on a bed using a pair of radar systems. The participants were three healthy males in their twenties. Each measurement time was 5 minutes.

Table 1 gives the TCR, AR, and RMSE in estimating the heart IBI using radar 1 (chest) and radar 2 (shoulder). For each participant, table cells for the body part (chest or shoulder) with a higher accuracy are filled in gray. For all participants, the TCR was higher when measuring the shoulder than when measuring the chest. The difference in TCRs when measuring the shoulder and chest was $11 \%$, $10 \%$, and $30 \%$ for participants $\mathrm{A}, \mathrm{B}$, and $\mathrm{C}$, respectively. The AR for the shoulder was higher than that for the chest by $16 \%$ and $31 \%$ for participants $\mathrm{A}$ and $\mathrm{C}$, respectively, whereas the ARs for the chest and shoulder were almost the same for participant B. The TCR and AR are different for different participants, indicating that individual differences cannot be ignored.

The difference in accuracies can be partly explained by the shapes of the body parts. In general, the shape of a human chest is more complicated than that of a shoulder, and thus, the radar signal from the chest contains more overlapping and interfering echoes, leading to low accuracy. Even if our radar systems receive multiple echoes from different body parts, as long as the difference in distances to the reflecting parts is greater than the range resolution $(75 \mathrm{~mm})$, the echoes can be separated and the heart IBI can be measured accurately. For this reason, the echo from the shoulder was not affected so much by interference from other body parts, such as the head and torso.

Figures 4-9 show the heart IBI estimated from data of the ECG (black line) and radar (circles) for each participant. The red and blue circles respectively show accurate and inaccurate estimates made from radar signals; estimate points with error of less than $\varepsilon=50 \mathrm{~ms}$ are plotted in red. As explained in the previous section, the RMSE is calculated for the red plots only. 


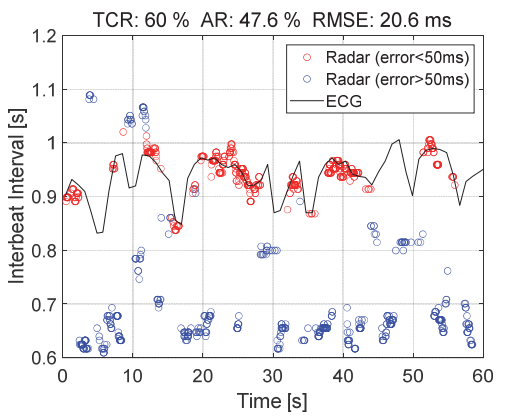

Fig. 4 Heart IBI estimated using the radar echo from participant A's chest; accurate radar (red circle), inaccurate radar (blue circle), and reference ECG (black line).

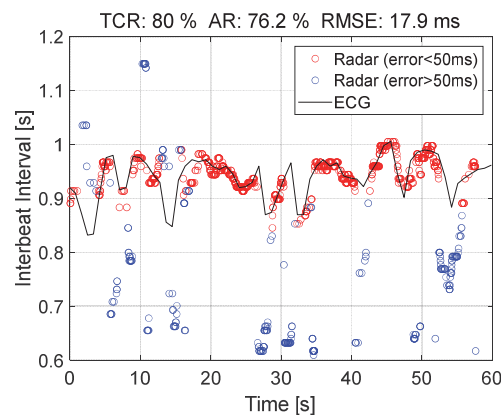

Fig. 5 Heart IBI estimated using the radar echo from participant A's shoulder; accurate radar (red circle), inaccurate radar (blue circle), and reference ECG (black line).

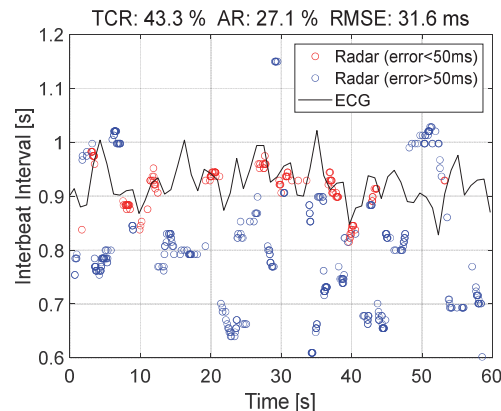

Fig. 6 Heart IBI estimated using the radar echo from participant B's chest; accurate radar (red circle), inaccurate radar (blue circle), and reference ECG (black line).

\section{Experiment Simulating Sleep with Body Motion}

We next conducted an experiment with the same three participants rolling over on a bed. Each participant was measured for 3 minutes using the same pair of radar systems installed at the same positions as in the previous section. The timetable of the measurement is summarized in Table 2. Each participant was instructed to roll over twice during the measurement. Participants were first in a right lateral recumbent position for 1 minute, then rolled over and remained in a supine position for another minute, and then rolled over again and stayed in a left lateral recumbent position for an-

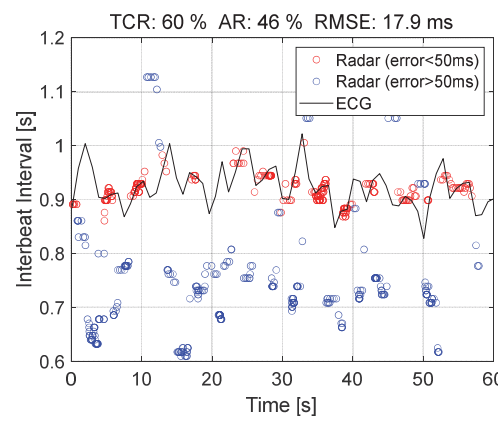

Fig. 7 Heart IBI estimated using the radar echo from participant B's shoulder; accurate radar (red circle), inaccurate radar (blue circle), and reference ECG (black line).

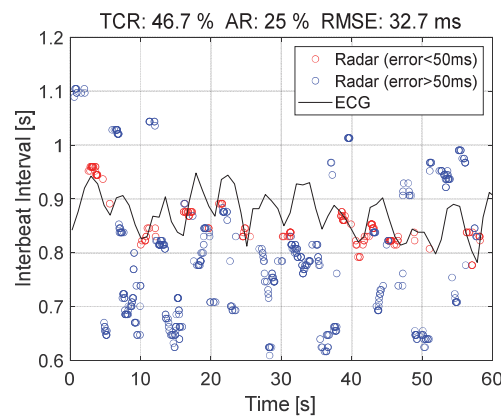

Fig. 8 Heart IBI estimated using the radar echo from participant C's chest; accurate radar (red circle), inaccurate radar (blue circle), and reference ECG (black line).

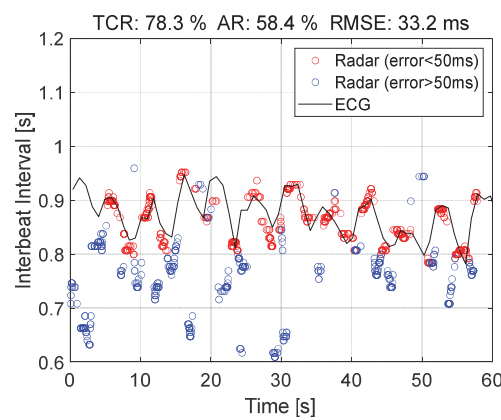

Fig.9 Heart IBI estimated using the radar echo from participant C's shoulder; accurate radar (red circle), inaccurate radar (blue circle), and reference ECG (black line).

Table 2 Timetable of the participants' position and body part measured.

\begin{tabular}{|c|c|c|c|}
\hline Time & Position & Radar 1 & Radar 2 \\
\hline $\begin{array}{c}\text { Time slot T1 } \\
0 \leq t<60 \mathrm{~s}\end{array}$ & $\begin{array}{c}\text { Right lateral } \\
\text { recumbent }\end{array}$ & $\begin{array}{c}\text { Left } \\
\text { shoulder }\end{array}$ & Back \\
\hline $\begin{array}{c}\text { Time slot T2 } \\
60 \mathrm{~s} \leq t<120 \mathrm{~s}\end{array}$ & Supine & Chest & $\begin{array}{c}\text { Left } \\
\text { shoulder }\end{array}$ \\
\hline $\begin{array}{c}\text { Time slot T3 } \\
120 \mathrm{~s} \leq t<180 \mathrm{~s}\end{array}$ & $\begin{array}{c}\text { Left lateral } \\
\text { recumbent }\end{array}$ & $\begin{array}{c}\text { Right } \\
\text { shoulder }\end{array}$ & Chest \\
\hline
\end{tabular}

other minute.

Figures 10 and 11 show an estimated body displacement $d(t)$ (black) and an estimated range $r_{0}(t)($ red). Black vertical lines indicate the times when body motion was detected 


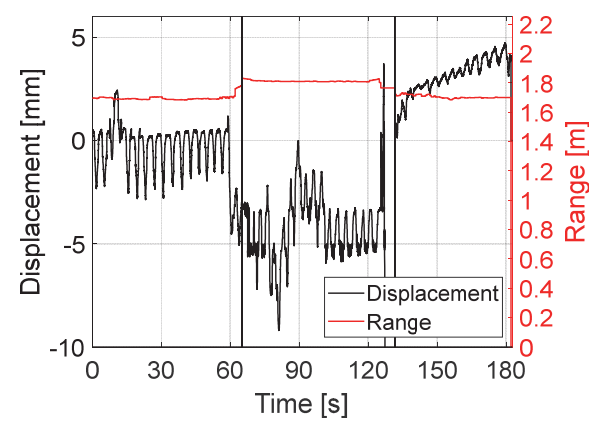

Fig. 10 Body displacement $\boldsymbol{d}(\boldsymbol{t})$ (black) and range $\boldsymbol{r}_{0}(\boldsymbol{t})$ (red) estimated for participant A using radar 1.

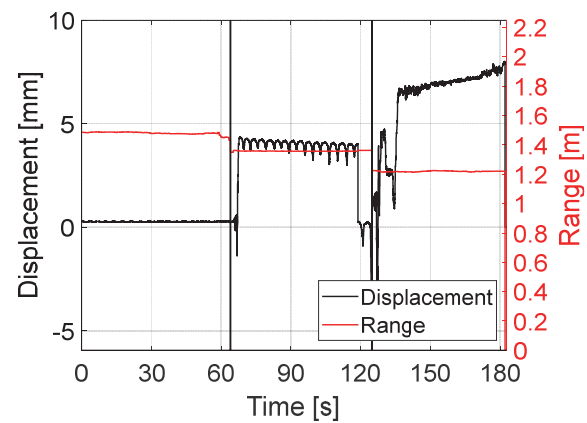

Fig. 11 Body displacement $\boldsymbol{d}(\boldsymbol{t})$ (black) and range $\boldsymbol{r}_{0}(\boldsymbol{t})$ (red) estimated for participant A using radar 2.

using the proposed method. It is seen that the estimated range $r_{0}(t)$ changes at the two instances of rolling over. We also see a quasi-periodic change in the body displacement $d(t)$ resulting from respiration. In the figures, the two body movements were correctly detected.

In Fig. 11, in the time range $0 \leq t \leq 60 \mathrm{~s}$, the body displacement $d(t)$ is small. By contrast, for $160 \mathrm{~s} \leq t \leq 180 \mathrm{~s}$, the participant was in the left lateral recumbent position, where his left arm was shadowing his chest, resulting in severe interference and nonperiodic displacement $d(t)$. Figures 12-14 show the heart IBI of participant A estimated using radar 1 while Figs. 15-17 show the heart IBI of the same participant estimated using radar 2. Using radar 1, it is seen that the IBI in T3 (Fig. 14) is more accurate than that in T1 and that in T2 (Figs. 12 and 13). Participant A was not immediately below radar 1 but shifted laterally in $\mathrm{T} 1$ according to our video recording. Therefore, the measured echo in T1 was not reflected from his right shoulder but from the diagonal backside of his torso, which lowered the accuracy in T1. Using radar 2, the IBI in T2 (Fig. 16) was the most accurate among those in the three time slots (Figs. 15-17) because the shoulder of the participant was facing radar 2 in $\mathrm{T} 2$.

Tables 3, 4, and 5 give the performance indices of heart IBI estimation for all participants. In the tables, the cells for a body part (chest or shoulder) having better performance are filled in gray. The results are consistent for all participants with a few exceptions. In T1, the performance of radar 1 is higher than that of radar 2 with a few exceptions. Radar 2
Table 3 Heart IBI estimation performance for participant A.

\begin{tabular}{|c|c|c|c|c|}
\hline Time slot & Radar & TCR & AR & RMSE \\
\hline \multirow{2}{*}{ T1 } & 1 & $48 \%$ & $25 \%$ & $28.5 \mathrm{~ms}$ \\
\cline { 2 - 5 } & 2 & $45 \%$ & $19 \%$ & $22.5 \mathrm{~ms}$ \\
\hline \multirow{2}{*}{$\mathrm{T} 2$} & 1 & $45 \%$ & $26 \%$ & $40.2 \mathrm{~ms}$ \\
\cline { 2 - 5 } & 2 & $90 \%$ & $90 \%$ & $34.0 \mathrm{~ms}$ \\
\hline \multirow{2}{*}{$\mathrm{T} 3$} & 1 & $85 \%$ & $79 \%$ & $20.7 \mathrm{~ms}$ \\
\cline { 2 - 5 } & 2 & $43 \%$ & $27 \%$ & $30.7 \mathrm{~ms}$ \\
\hline
\end{tabular}

Table 4 Heart IBI estimation performance for participant B.

\begin{tabular}{|c|c|c|c|c|}
\hline Time slot & Radar & TCR & AR & RMSE \\
\hline \multirow{2}{*}{ T1 } & 1 & $43 \%$ & $29 \%$ & $29.5 \mathrm{~ms}$ \\
\cline { 2 - 5 } & 2 & $32 \%$ & $17 \%$ & $24.2 \mathrm{~ms}$ \\
\hline \multirow{2}{*}{$\mathrm{T} 2$} & 1 & $23 \%$ & $21 \%$ & $31.8 \mathrm{~ms}$ \\
\cline { 2 - 5 } & 2 & $55 \%$ & $27 \%$ & $17.5 \mathrm{~ms}$ \\
\hline \multirow{2}{*}{$\mathrm{T} 3$} & 1 & $63 \%$ & $61 \%$ & $22.5 \mathrm{~ms}$ \\
\cline { 2 - 5 } & 2 & $40 \%$ & $28 \%$ & $30.5 \mathrm{~ms}$ \\
\hline
\end{tabular}

Table 5 Heart IBI estimation performance for participant C.

\begin{tabular}{|c|c|c|c|c|}
\hline Time slot & Radar & TCR & AR & RMSE \\
\hline \multirow{2}{*}{$\mathrm{T} 1$} & 1 & $62 \%$ & $51 \%$ & $27.1 \mathrm{~ms}$ \\
\cline { 2 - 5 } & 2 & $87 \%$ & $67 \%$ & $18.2 \mathrm{~ms}$ \\
\hline \multirow{2}{*}{$\mathrm{T} 2$} & 1 & $53 \%$ & $38 \%$ & $25.6 \mathrm{~ms}$ \\
\cline { 2 - 5 } & 2 & $87 \%$ & $79 \%$ & $24.0 \mathrm{~ms}$ \\
\hline \multirow{2}{*}{$\mathrm{T} 3$} & 1 & $63 \%$ & $49 \%$ & $21.7 \mathrm{~ms}$ \\
\cline { 2 - 5 } & 2 & $35 \%$ & $23 \%$ & $27.5 \mathrm{~ms}$ \\
\hline
\end{tabular}

outperforms radar 1 in T2 while radar 1 outperforms radar 2 in T3. It is concluded from these results that installing multiple radar systems at different places is advantageous in constant sleep monitoring because the orientation of the target person cannot be predicted.

\section{Overnight Radar Measurement of a Sleeping Person}

We finally measured a participant who slept in a bed overnight so that we could evaluate the performance of the system with realistic data. The experimental setup was the same as that adopted in the previous section. We recorded the participant using an infrared video camera. The measurement time was 500 minutes. Table 6 summarizes the position of the participant, which was obtained manually from the infrared video recording.

The upper, middle, and lower figures of Fig. 18 respectively show the body displacement $d(t)$ obtained using radar 1 , radar 2, and both radar systems. The estimated body movements are shown as black vertical lines, whereas the actual body movements extracted manually from the video footage are shown as red vertical lines.

When using only a single radar system, false body movements were erroneously detected; the number of detected movements were 63 and 101 when using radars 1 and 2 , respectively (as shown by black vertical lines in the upper and middle panels in Fig. 18). Among them, only 10 movements were confirmed manually in the video recording 


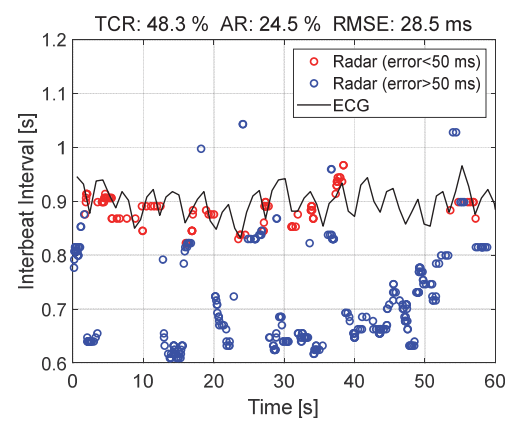

Fig. 12 Heart IBI of participant A in time slot T1 estimated using reference ECG (black line) and radar 1 (red and blue circles); accurate estimates are in red while inaccurate estimates are in blue.

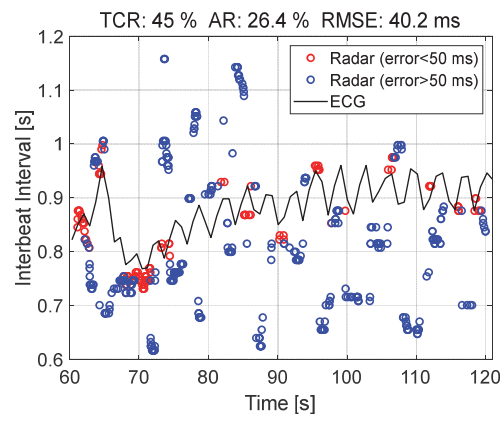

Fig. 13 Heart IBI of participant A in time slot T2 estimated using the reference ECG (black line) and radar 1 (red and blue circles); accurate estimates are in red while inaccurate estimates are in blue.

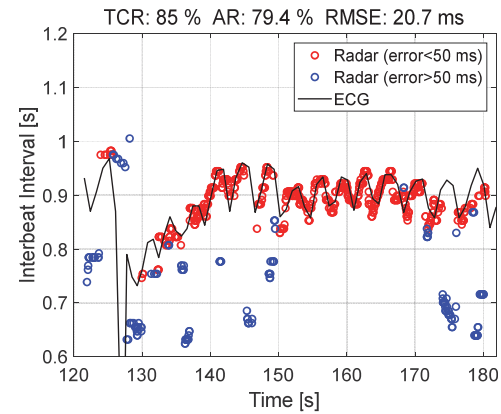

Fig. 14 Heart IBI of participant A in time slot T3 estimated using the reference ECG (black line) and radar 1 (red and blue circles); accurate estimates are in red while inaccurate estimates are in blue.

(as shown by red short vertical lines). By contrast, 12 movements were detected by the two radars simultaneously, where simultaneous detection is defined as the two radar systems detecting a movement with a time difference less than $6 \mathrm{~s}$ (as shown by the black vertical lines in the lower panel of Fig. 18). As a result, seven movements (M1, M4, M5, M6, M7, M9, and M10) out of a total of 10 movements (Table 6) were correctly detected. Here, the detection performance is evaluated in terms of its precision and recall. When radars 1 and 2 were used separately, the precisions were 10/63= $15.9 \%$ and $10 / 101=9.9 \%$, respectively; the recalls were both $8 / 10=80 \%$. By contrast, when using both radars, the precision was $7 / 12=58.3 \%$, and the recall was $7 / 10=70 \%$,

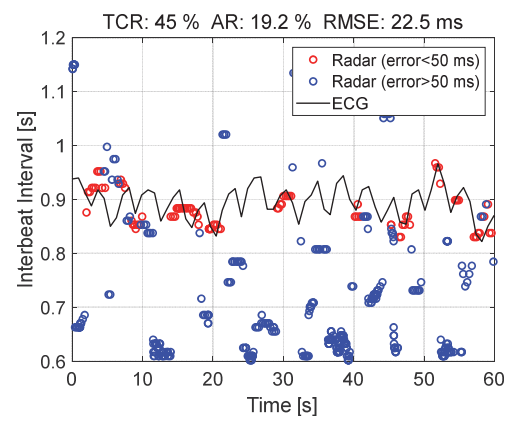

Fig. 15 Heart IBI of participant A in time slot T1 estimated using reference ECG (black line) and radar 2 (red and blue circles); accurate estimates are in red while inaccurate estimates are in blue.

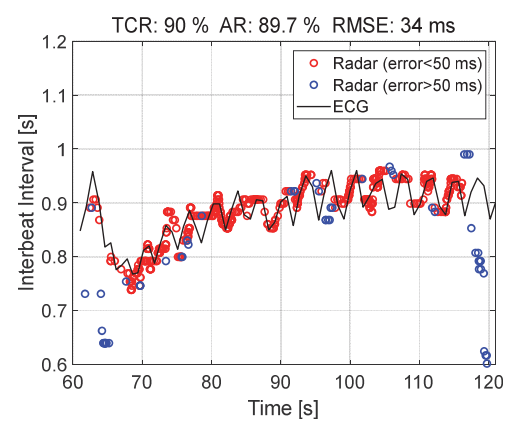

Fig. 16 Heart IBI of participant A in time slot T2 estimated using reference ECG (black line) and radar 2 (red and blue circles); accurate estimates are in red while inaccurate estimates are in blue.

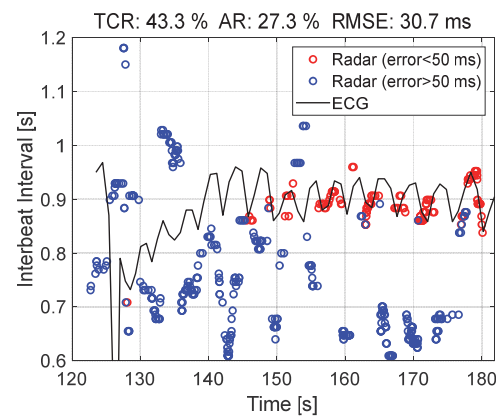

Fig. 17 Heart IBI of participant A in time slot T3 estimated using reference ECG (black line) and radar 2 (red and blue circles); accurate estimates are in red while inaccurate estimates are in blue.

which suggests that the recall decreased by $10 \%$, whereas the precision increased by $42.4 \%$ and $48.4 \%$.

In addition to the movements listed in Table 6, the measurement system detected five smaller limb movements without the participant rolling over at $t=240.8,316.1$, 329.0, 344.9 , and $367.3 \mathrm{~min}$, which was confirmed by manually checking the video recording; the two radar systems detected these five movements at the same time. Although these limb motions are relatively small and do not change the torso orientation, such motions can still interfere with the recording of the vital signs, reducing the accuracy. The proposed method detects such small body movements, which is an important function that improves the overall accuracy of 
Table 6 Lapse time and position of the participant.

\begin{tabular}{|c|c|c|}
\hline Movement & $\begin{array}{c}\text { Time } \\
{[\mathrm{min}]}\end{array}$ & End position \\
\hline M0 & 0 & Supine \\
\hline M1 & 212.4 & Left lateral recumbent \\
\hline M2 & 227.8 & Supine \\
\hline M3 & 321.0 & Right lateral recumbent \\
\hline M4 & 332.3 & Supine \\
\hline M5 & 351.5 & Left lateral recumbent \\
\hline M6 & 360.5 & Supine \\
\hline M7 & 363.5 & Left lateral recumbent \\
\hline M8 & 373.1 & Supine \\
\hline M9 & 382.3 & Left lateral recumbent \\
\hline M10 & 387.4 & Supine \\
\hline
\end{tabular}
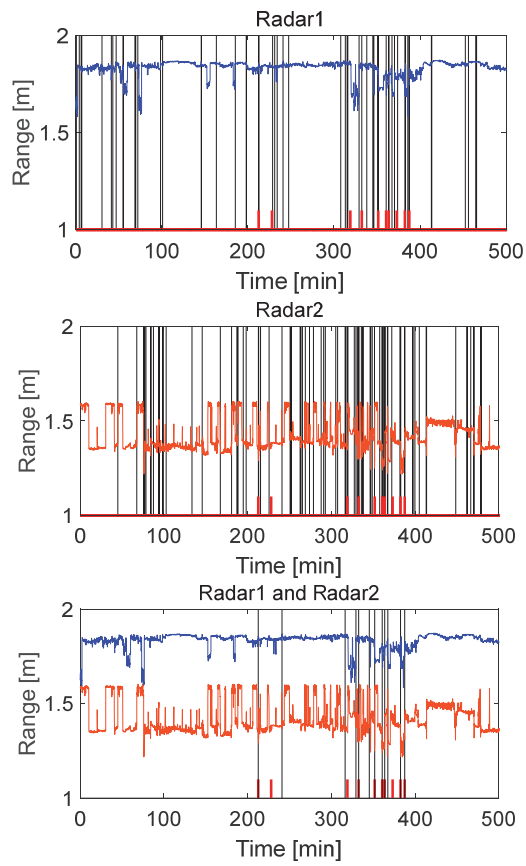

Fig. 18 Target range (blue and orange) estimated using radar 1 (upper), radar 2 (middle), and both radar systems (lower). The data from radars 1 and 2 are displayed in blue and orange. The body movements were estimated using radar (black lines) while reference body movements were extracted manually from the infrared video recording. (red lines).

the noncontact measurement of vital signs.

Figure 19 shows the TCR (top) and AR (bottom) in estimating the heart IBI using radar 1 (blue) and radar 2 (orange). Radar 2 was more accurate than radar 1 when the participant was in the supine position. Although the time duration while the participant was in a lateral recumbent position was comparatively short, when the lateral recumbent position was confirmed in the video, radar 1 was more accurate than radar 2 in terms of the TCR and AR. These results are consistent with the results presented in previous sections, indicating that the radar measurement of the shoulder is more suitable than that of the chest. The average TCR was $39.3 \%$ and $47.8 \%$ for radars 1 and 2, respectively. The average AR was $26.6 \%$ and $30.8 \%$ for radars 1 and 2 , respectively.
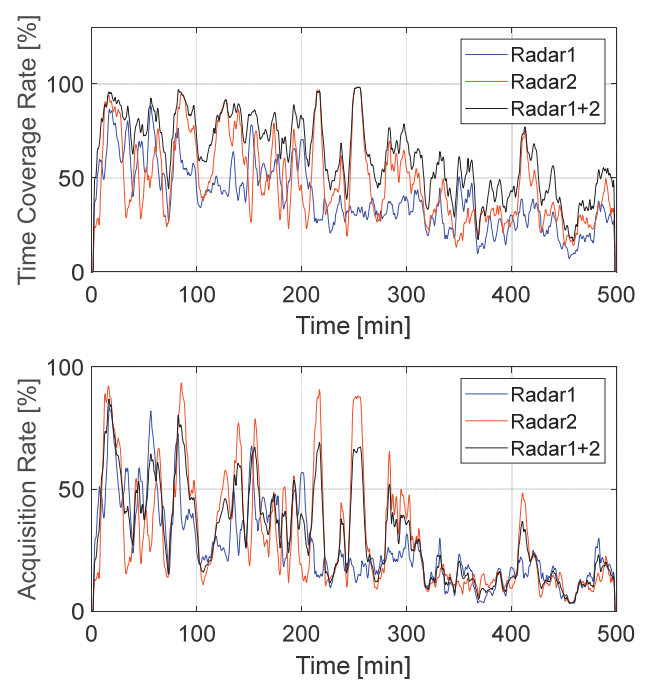

Fig. 19 Time coverage rate (top) and acquisition rate (bottom) when using radar 1 (blue), radar 2 (orange) and both radars 1 and 2 (black).

To demonstrate the effectiveness of using the two radars together, the IBI estimates from radars 1 and 2 were combined to evaluate the TCR and AR values, which are shown in black in the upper and lower graphs in Fig. 19, respectively. Because the IBI estimates are simply combined, the total number of estimates is the summation of those from radars 1 and 2. Therefore, the AR of the combined IBI (lower panel of Fig. 19) is between the ARs for radars 1 and 2. By contrast, the TCR of the combined IBI (upper panel of Fig. 19) is always higher than any of the ARs from radars 1 and 2. This is because, when using two radars, the shoulder of the participant is more likely to be facing one of the radars than it is when using only a single radar. When the IBIs from the two radars were combined, the average TCR and AR were $62.7 \%$ and $30.7 \%$, respectively. The TCR of the combined IBI was higher than the TCRs of radars 1 and 2 by $23.4 \%$ and $14.9 \%$, respectively.

\section{Conclusion}

We demonstrated the effectiveness of using a pair of MMW UWB MIMO array radar systems in monitoring the heartbeat and body movements of a sleeping person. We conducted three types of measurements to evaluate the performance of the system. In the first and second experiments, participants were instructed to lie down on a bed with or without rolling over; in the third experiment, a participant slept overnight. The heart IBIs estimated from radar data were evaluated carefully in terms of three indices that were proposed in this paper. It was shown that the location of the radar affected the accuracy of the noncontact measurement of the heartbeat and body movement during sleep. The use of multiple radar systems was therefore demonstrated to be effective in observing the target person from different angles and monitoring the heartbeat constantly and accurately. It was confirmed that the radar measurement of the shoulder can outperform that of the chest for a sleeping person in estimating the heart IBI. In 
addition, body movements can be detected more accurately using a pair of radar systems than using a single-radar system. These experiments and the careful performance evaluation demonstrated that using a pair of radar systems is advantageous in monitoring a sleeping person and measuring the vital signs and body movements with high accuracy. Although our monitoring system comprises two radar systems, it is inferred that using more than two radar systems would be even more beneficial in constantly monitoring a sleeping person.

\section{Acknowledgments}

We thank Mr. Hiroyuki Sakai, Mr. Kenji Mizutani, Mr. Takeshi Fukuda, and Mr. Kenichi Inoue of Panasonic Corporation, Kadoma, Osaka, for their technical assistance; Dr. Shigeaki Okumura of MaRI Co. Ltd., Kyoto, Japan for his help with radar measurements; and Glenn Pennycook, MSc, from Edanz Group (www.edanzediting.com/ac) for editing a draft of this manuscript. This study was supported in part by JST JPMJPR1873, JST COI JPMJCE1307, JSPS KAKENHI $19 \mathrm{H} 02155$.

\section{References}

[1] T. Sakamoto, R. Imasaka, H. Taki, T. Sato, M. Yoshioka, K. Inoue, T. Fukuda, and H. Sakai, "Feature-based correlation and topological similarity for interbeat interval estimation using ultrawideband radar," IEEE Trans. Biomed. Eng., vol.63, no.4, pp.747-757, 2016.

[2] T. Sakamoto, R. Imasaka, H. Taki, T. Sato, M. Yoshioka, K. Inoue, T. Fukuda, and H. Sakai, "Accurate heartbeat monitoring using ultra-wideband radar," IEICE Electron. Express, vol.12, no.3, pp.20141197, 2015.

[3] A.D. Droitcour, O. Boric-Lubecke, V.M. Lubecke, J. Lin, and G.T. A. Kovacs, "Range correlation and I/Q performance benefits in singlechip silicon Doppler radars for noncontact cardiopulmonary monitoring,” IEEE Trans. Microw. Theory Techn., vol.52, no.3, pp.838-848, 2004

[4] M. Chen, O. Boric-Lubecke, and V.M. Lubecke, "0.5- $\mu$ m CMOS implementation of analog heart-rate extraction with a robust peak detector," IEEE Trans. Instrum. Meas., vol.57, no.4, pp.690-698, 2008

[5] W. Massagram, V.M. Lubecke, A. Høst-Madsen, and O. BoricLubecke, "Assessment of heart rate variability and respiratory sinus arrhythmia via Doppler radar," IEEE Trans. Microw. Theory Techn., vol.57, no.10, pp.2542-2549, 2009.

[6] S.D. Min, J.K. Kim, H.S. Shin, Y.H. Yun, C.K. Lee, and M. Lee, "Noncontact respiration rate measurement system using an ultrasonic proximity sensor," IEEE Sensors J., vol.10, no.11, pp.1732-1739, 2010

[7] J. Kranjec, S. Begus, J. Drnovsek, and G. Gersak, "Novel methods for noncontact heart rate measurement: A feasibility study," IEEE Trans. Instrum. Meas., vol.63, no.4, pp.838-847, 2014.

[8] W. Hu, Z. Zhao, Y. Wang, H. Zhang, and F. Lin, "Noncontact accurate measurement of cardiopulmonary activity using a compact quadrature Doppler radar sensor," IEEE Trans. Biomed. Eng., vol.61, no.3, pp.725-735, 2014.

[9] K. Sasaki, N. Honma, T. Nakayama, and S. Iizuka, "RSSI-based living-body radar using single RF front-end and tunable parasitic antennas," IEICE Trans. Commun., vol.E101-B, no.2, pp.392-399, Feb. 2018.

[10] F. Zhu, K. Wang, and K. Wu, "A fundamental-and-harmonic dualfrequency Doppler radar system for vital signs detection enabling radar movement self-cancellation," IEEE Trans. Microw. Theory Techn., vol.66, no.11, pp.5106-5118, 2018.

[11] S. Izumi, T. Okano, D. Matsunaga, H. Kawaguchi, and M. Yoshimoto, "Non-contact instantaneous heart rate extraction system using 24-GHz microwave Doppler sensor," IEICE Trans. Commun., vol.E102-B, no.6, pp.1088-1096, June 2019.

[12] C. Ye, K. Toyoda, and T. Ohtsuki, "A stochastic gradient approach for robust heartbeat detection with Doppler radar using timewindow-variation technique," IEEE Trans. Biomed. Eng., vol.66, no.6, pp.1730-1741, 2019 .

[13] D. Nagae and A. Mase, "Measurement of heart rate variability and stress evaluation by using microwave reflectometric vital signal sensing," Rev. Sci. Instrum., vol.81, no.9, pp.094301, 2010.

[14] T. Sakamoto, S. Okumura, R. Imanishi, H. Taki, T. Sato, M. Yoshioka, K. Inoue, T. Fukuda, and H. Sakai, "Remote heartbeat monitoring from human soles using $60-\mathrm{GHz}$ ultra-wideband radar," IEICE Electron. Express, vol.12, no.21, pp.20150786, Oct. 2015.

[15] T. Sakamoto, M. Muragaki, K. Tamura, S. Okumura, T. Sato, K. Mizutani, K. Inoue, T. Fukuda, and H. Sakai, "Measurement of instantaneous heart rate using radar echoes from the human head," Electron. Lett., vol.54, no.14, pp.864-866, 2018.

[16] G. Vinci, S. Lindner, F. Barbon, S. Mann, M. Hofmann, A. Duda, R. Weigel, and A. Koelpin, "Six-port radar sensor for remote respiration rate and heartbeat vital-sign monitoring," IEEE Trans. Microw. Theory Techn., vol.61, no.5, pp.2093-2100, 2013.

[17] F.-K. Wang, T.-S. Horng, K.-C. Peng, J.-K. Jau, J.-Y. Li, and C.-C. Chen, "Single-antenna Doppler radars using self and mutual injection locking for vital sign detection with random body movement cancellation," IEEE Trans. Microw. Theory Techn., vol.59, no.12, pp.3577-3587, 2011

[18] M.-C. Tang, F.-K. Wang, and T.-S. Horng, "Single self-injectionlocked radar with two antennas for monitoring vital signs with large body movement cancellation," IEEE Trans. Microw. Theory Techn., vol.65, no.12, pp.5324-5333, 2017.

[19] C. Li, Y. Xiao, and J. Lin, "Experiment and spectral analysis of a low-power Ka-band heartbeat detector measuring from four sides of a human body," IEEE Trans. Microw. Theory Techn., vol.54, no.12, pp.4464-4471, 2006.

[20] J. Wang, X. Wang, Z. Zhu, J. Huangfu, C. Li, and L. Ran, "1-D microwave imaging of human cardiac motion: An ab-initio investigation," IEEE Trans. Microw. Theory Techn., vol.61, no.5, pp.21012107, 2013.

[21] J.E. Kiriazi, O. Boric-Lubecke, and V.M. Lubecke, "Dual-frequency technique for assessment of cardiopulmonary effective RCS and displacement,” IEEE Sensors J., vol.12, no.3, pp.574-582, 2012.

[22] F. Lin, Y. Zhuang, C. Song, A. Wang, Y. Li, C. Gu, C. Li, and W. $\mathrm{Xu}$, "SleepSense: A noncontact and cost-effective sleep monitoring system,” IEEE Trans. Biomed. Circuits Syst., vol.11, no.1, pp.189202, 2017.

[23] L. Zhang, J. Xiong, H. Zhao, H. Hong, X. Zhu, and C. Li, "Sleep stages classification by CW Doppler radar using bagged trees algorithm," Proc. 2017 IEEE Radar Conf., Seattle, WA, 2017. DOI: 10.1109/RADAR.2017.7944310

[24] H. Hong, L. Zhang, C. Gu, Y. Li, G. Zhou, and X. Zhu, "Noncontact sleep stage estimation using a CW Doppler radar," IEEE J. Emerg. Sel. Topics Circuits Syst., vol.8, no.2, pp.260-270, 2018.

[25] M. Baboli, A. Singh, B. Soll, O. Boric-Lubecke, and V.M. Lubecke, "Wireless sleep apnea detection using continuous wave quadrature Doppler radar," IEEE Sensors J., vol.20, no.1, pp.538-545, 2020.

[26] T. Miwa, S. Ogiwara, and Y. Yamakoshi, "MIMO radar system for respiratory monitoring using $\mathrm{Tx}$ and $\mathrm{Rx}$ modulation with M-sequence codes," IEICE Trans. Commun., vol.E93-B, no.9, pp.2416-2423, Sept. 2010

[27] A.B. Tataraidze, L.N. Anishchenko, L.S. Korostovtseva, M.V Bochkarev, and Y.V. Sviryaev, "Non-contact respiratory monitoring of subjects with sleep-disordered breathing," Proc. 2018 IEEE Int'l Conf. Quality Management, Transport and Information Secu- 
rity, Information Technologies, St. Petersburg, pp.736-738, 2018. DOI: 10.1109/ITMQIS.2018.8525001

[28] S. Kang, Y.-H. Lim, Y. Lee, J. Choi, H.-K. Park, S.H. Cho, and S.H. Cho, "Comparison of sleep parameter with actigraphy and IRUWB sensor based on Polysomnography," Proc. 2018 Int'l Conf. Network Infrastructure and Digital Content, Guiyang, pp.320-323, 2018. DOI: 10.1109/ICNIDC.2018.8525833

[29] Y. Cheng, Z. Yang, D. Hong, and J. Chen, "Sleep anomaly events detection based on adaptive double threshold CFAR detector using UWB radar," Proc. 2019 IEEE Int'l Conf. Signal Processing, Commun. and Computing, Dalian, 2019.

[30] J.M. Lee, J.W. Choi, and S.H. Cho, "Movement analysis during sleep using an IR-UWB radar sensor," Proc. 2016 IEEE Int'l Conf. Network Infrastructure and Digital Content, Beijing, 2016. DOI: 10.1109/ICNIDC.2016.7974622

[31] V.P. Tran and A.A. Al-Jumaily, "Non-contact Doppler radar based prediction of nocturnal body orientations using deep neural network for chronic heart failure patients," Proc. 2017 Int'l Conf. Electrical and Computing Technologies and Applications, Ras Al Khaimah, 2017. DOI: $10.1109 /$ ICECTA.2017.8252020

[32] N. Du, K. Liu, L. Ge, and J. Zhang, "ApneaRadar: A $24 \mathrm{GHz}$ radarbased contactless sleep apnea detection system," Proc. 2017 2nd Int'l Conf. Frontiers of Sensors Technologies, Shenzhen, 2017. DOI: 10.1109/ICFST.2017.8210539

[33] T. Sakamoto and K. Yamashita, "Noncontact measurement of autonomic nervous system activities based on heart rate variability using ultra-wideband array radar," IEEE J. Electromagn., RF, Microw. Med. Biol., vol.4, no.3, pp.208-215, 2020. DOI 10.1109/JERM.2019.2948827

[34] S. Suzuki, T. Matsui, M. Kagawa, T. Asao, and K. Kotani, "An approach to a non-contact vital sign monitoring using dual-frequency microwave radars for elderly care," J. Biomedical Science and Engineering, vol.6, no.7, pp.704-711, 2013.

[35] A.Q. Javaid, C.M. Noble, R. Rosenberg, and M.A. Weitnauer, "Towards sleep apnea screening with an under-the-mattress IR-UWB radar using machine learning," Proc. 2015 IEEE 14th Int'1 Conf. Machine Learning and Applications, Miami, FL, 2015. DOI: 10.1109/ ICMLA.2015.79

[36] M. Kagawa, K. Suzumura, and T. Matsui, "Sleep stage classification by non-contact vital signs indices using Doppler radar sensors," Proc. 2016 38th Annual Int'l Conf. IEEE Engineering in Med. Biology Soc., Orlando, FL, 2016. DOI: 10.1109/EMBC.2016.7591829

[37] K. Higashi, G. Sun, and K. Ishibashi, "Precise heart rate measurement using non-contact Doppler radar assisted by machine-learning-based sleep posture estimation," Proc. 2019 41st Annual Int'l Conf. IEEE Engineering in Medicine and Biology Society, Berlin, 2019. DOI: 10.1109/EMBC.2019.8857830

[38] S. Okumura, T. Sakamoto, T. Sato, M. Yoshioka, K. Inoue, T. Fukuda, and H. Sakai, "Comparison of clutter rejection techniques for measurement of small displacements of body surface using radar," Electron. Lett., vol.52, no.19, pp.1635-1637, 2016.

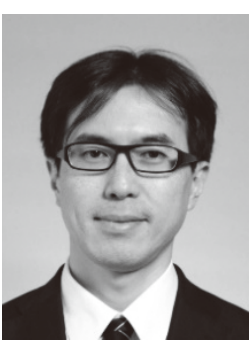

Takuya Sakamoto received a B.E. degree in electrical and electronic engineering from Kyoto University, Kyoto, Japan, in 2000 and M.I. and $\mathrm{Ph} . \mathrm{D}$. degrees in communications and computer engineering from the Graduate School of Informatics, Kyoto University, in 2002 and 2005, respectively. From 2006 through 2015, he was an Assistant Professor at the Graduate School of Informatics, Kyoto University. From 2011 to 2013, he was also a Visiting Researcher at the Delft University of Technology, Delft, the Netherlands. From 2015 to 2019, he was an Associate Professor at the Graduate School of Engineering, University of Hyogo, Himeji, Japan. In 2017, he was also a Visiting Scholar at the University of Hawaii at Manoa, Honolulu, HI, USA. From 2018, he has been a PRESTO Researcher of the Japan Science and Technology Agency, Kawaguchi, Japan. Currently, he is an Associate Professor at the Department of Electrical Engineering, Graduate School of Engineering, Kyoto University. His current research interests are system theory, inverse problems, radar signal processing, radar imaging, and the wireless sensing of vital signs.

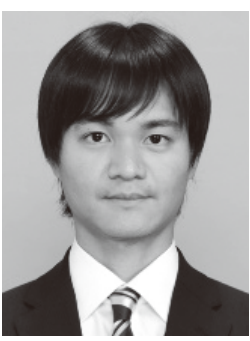

Sohei Mitani received a B.E. degree in electrical and electronic engineering from Kyoto University, Kyoto, Japan, in 2016, and an M.I. degree in communications and computer engineering from the Graduate School of Informatics, Kyoto University in 2018. His research interests include radar signal processing and the noncontact measurement of vital signs.

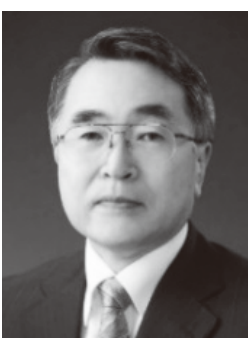

Toru Sato received B.E., M.E., and Ph.D. degrees in electrical engineering from Kyoto University, Kyoto, Japan in 1976, 1978, and 1982 , respectively. He has been with Kyoto University since 1983 and is Vice Director and Professor at the Institute for Liberal Arts and Sciences. His major research interests include the system design and signal processing aspects of UWB radars, atmospheric radars, radar remote sensing of the atmosphere, and biomedical imaging. $\mathrm{He}$ is a fellow of the Institute of Electronics, Information, and Communication Engineers of Japan and a member of the Society of Geomagnetism and Earth, Planetary and Space Sciences, the Institute of Electrical Engineers of Japan, and the Institute of Electrical and Electronics Engineers. 